\title{
LOFT, the Large Observatory For X-ray Timing
}

\section{E. Bozzo*}

ISDC Data Centre for Astrophysics, Chemin d'Ecogia 16, CH-1290 Versoix, Switzerland;

E-mail: enrico.bozzo@unige.ch

\section{J. W. den Herder}

SRON, the Netherlands Institute for Space Research, Sorbonnelaan 2, 3584 CA Utrecht, the Netherlands

M. Feroci

INAF/IASF, Via del Fosso del Cavaliere 100, I-00133 Roma, Italy

\section{Stella}

INAF-OAR, Via di Frascati 33, I-00040 Monteporzio Catone, Italy

\section{on the behalf of the LOFT consortium}

The Large Observatory For X-ray Timing, LOFT, was selected by the European Space Agency as one of the four Cosmic Vision M3 candidate missions to compete for a launch opportunity at the start of the 2020s. Thanks to an innovative design and the development of large-area monolithic silicon drift detectors, the Large Area Detector (LAD) on board LOFT will operate in the 2-30 $\mathrm{keV}$ range (up to $50 \mathrm{keV}$ in expanded mode), and achieve an effective area of $\sim 10 \mathrm{~m}^{2}$ at $8 \mathrm{keV}$, a time resolution of $\sim 10 \mu \mathrm{s}$, and a spectral resolution of $\sim 260 \mathrm{eV}$ (FWHM at $6 \mathrm{keV}$ ). These characteristics make LOFT a perfectly suited instrument to perform high-time-resolution X-ray observations of collapsed objects in our galaxy and brightest supermassive black holes in active galactic nuclei. LOFT will yield unprecedented information on strongly curved spacetimes and matter under extreme conditions of pressure and magnetic field strength, thus addressing two of the fundamental questions of the Cosmic Vision Theme "Matter under extreme conditions": does matter orbiting close to the event horizon follow the predictions of general relativity? What is the equation of state of matter in neutron stars?

The Extreme and Variable High Energy Sky - extremesky2011,

September 19-23, 2011

Chia Laguna (Cagliari), Italy

\footnotetext{
*Speaker.
} 


\section{Introduction}

Collapsed objects, like neutron stars (NS) and black holes (BH), are characterized by exceptionally intense gravitational and magnetic fields. They provide a unique opportunity to reveal a variety of general relativistic effects and probe the conditions of matter in presence of supercritical magnetic fields and bulk densities exceeding those of atomic nuclei (Psaltis et al., 2008). Carrying out these investigations requires the exploitation of diagnostics that are capable of probing in situ matter motion in close vicinity of collapsed objects and/or measuring the rotation of the collapsed object itself. In this respect, both techniques based on X-ray spectroscopy and fast X-ray timing measurements are crucial. The former permit to reveal very broad profiles of X-ray emission lines (Fe K-shell in particular) from accretion disks extending down to the radius of the NSs and BHs marginally stable orbit. In these regions, velocities are comparable to the speed of light and well known relativistic effects (such as gravitational redshift, light-bending, frame-dragging, and Doppler shifts) distort the iron line shape into a characteristic broad shape. Measurements of the line profile then gives a detailed overview of the motion of matter very close to the central object. Among the available timing features, fast quasi periodic oscillations (QPOs) from BHs and NSs and coherent pulsations from the rotation of NSs and white dwarfs (WDs) are widely used to probe the intimate physical structure of these objects. QPOs in X-ray binaries and very massive BH in Active Galactic Nuclei (AGN), appear at the characteristic dynamical time-scales of the innermost disk regions, and are thus associated to the matter motion in strong field gravity. In the case of NSs, the detection and accurate measurement of coherent pulsations has been demonstrated effective in constraining its mass and radius, thus providing information on the NS equation of state (Stella et al., 2009; van der Klis et al., 2006; Strohmayer et al., 2006).

Many decisive progresses in all the above fields of research were made possible in particular through the exploitation of the time resolution and effective area of the proportional counters array (PCA, $\sim 0.67 \mathrm{~m}^{2}$ ) on-board RXTE (Jahoda et al., 1996). LOFT, the Large Observatory For Xray Timing, will constitute the next giant leap forward with respect to any existing X-ray timing devoted experiment, and it is being proposed as the natural follow-up to the more than 15 years of operations of RXTE. The instruments on-board LOFT will be endowed with an unprecedentedly large effective area $\left(\sim 10 \mathrm{~m}^{2}\right.$ at $\left.8 \mathrm{keV}\right)$ and a spectral resolution of $\sim 260 \mathrm{eV}$ (FWHM at $\left.6 \mathrm{keV}\right)$. This will revolutionise the research field of compact objects and yield a great opportunity to address many of the fundamental questions on the matter under extreme conditions that were left open by the previous generation of $\mathrm{X}$-ray instruments.

\section{The concept}

LOFT is specifically designed to exploit the diagnostics of rapid X-ray flux and spectral variability that directly probe the motion of matter down to distances very close to BHs and NSs. The successes achieved by RXTE in this context showed that a proper investigation of the energyresolved timing properties of the X-ray emission from cosmic sources requires the accurate measurement of the time-of-arrival and energy of the largest number of photons from the target source (Jahoda et al., 1996). The unambiguous identification of the target source in this type of experi-

ment can be achieved by narrowing the field of view by means of an aperture collimator, down to 

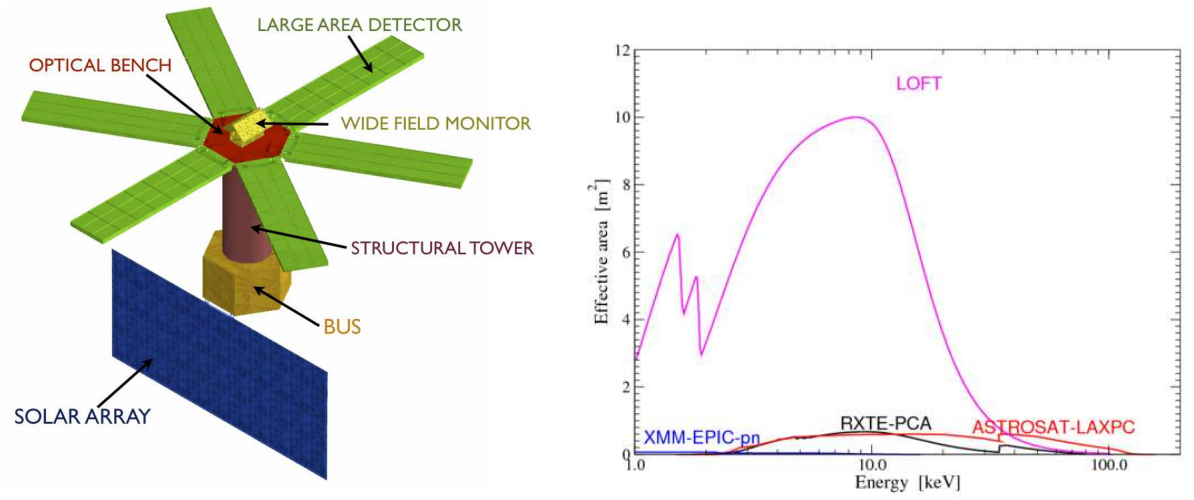

Figure 1: Left panel: Sketch of the LOFT satellite. The 6 deployable panels of the LAD, the WFM and the solar array are indicated. Right panel: effective area of the LOFT/LAD as a function of energy.

a level large enough (typically $\simeq 1^{\circ}$ ) to allow for pointing uncertainties yet small enough to reduce the aperture background (cosmic diffuse X-ray background) and the risk of source confusion. In this type of instruments, the knowledge of the impact point of the photon on the detector array is not needed (if not for the use of proper detector calibration data), so position sensitive detectors are not required. Instead, detector read-out segmentation is useful/necessary to reduce the effects of pile-up and dead time.

With these concepts in mind, the main instrument on-board LOFT (Feroci et al., 2011) was designed as a collimated experiment, operating in the 2-30 keV energy range and reaching an effective area of $\sim 10 \mathrm{~m}^{2}$ at $8 \mathrm{keV}$ and a spectral resolution of $\sim 260 \mathrm{eV}$ (FWHM) at $6 \mathrm{keV}$. This Large Area Detector (LAD) will provide a total of $\sim 140000 \mathrm{cts} / \mathrm{s}$ for a $500 \mathrm{mCrab}$ source, thus constituting a real breakthrough with respect to any other existing X-ray instrument (see Fig. 1). The LOFT scientific payload is completed by a coded-mask Wide Field Monitor (WFM) in charge of monitoring a large fraction of the sky potentially accessible to LAD, to provide the history and context for the sources observed by the LAD and trigger its observations on their most interesting states. The large field of view of the WFM will permit to observe in the same energy range of the LAD about $50 \%$ of the sky at once. The WFM is designed also to catch transient/bursting events down to a few mCrab fluxes and will provide for them data with a spectral resoution of $<500 \mathrm{eV}$ in the $2-50 \mathrm{keV}$ energy range and timing resolution of $10 \mu \mathrm{sec}$.

The LOFT satellite is considered to operate in a low equatorial earth orbit $\left(\sim 600 \mathrm{~km},<5^{\circ} \mathrm{deg}\right.$ inclination) in order to reduce the background and the radiation damage effect of South Atlantic Anomaly. The science return of the mission has been so far evaluated assuming a medium-small class and a Vega launcher, even though other options are being considered ${ }^{1}$.

\subsection{The Large area detector}

The Large Area Detector (LAD) of LOFT is designed as a classical collimated experiment. The key feature of the LAD design that allows reaching for the first time a very large effective area and a improved energy resolution is the low mass per unit area enabled by the solid-state

\footnotetext{
${ }^{1}$ See also the results of the ESA Concurrent Design Facility (CDF) at http://sci.esa.int/sciencee/www/object/index.cfm?fobjectid $=49357$
} 


\begin{tabular}{lll}
\multicolumn{3}{c}{ LAD } \\
\hline Parameter & Requirement & Goal \\
\hline Energy range & $2-30 \mathrm{keV}$ (nominal) & $1-30 \mathrm{keV}$ (nominal) \\
& $2-50 \mathrm{keV}$ (expanded) & $1-50 \mathrm{keV}$ (expanded) \\
Eff. area & $10.0 \mathrm{~m}^{2}(8 \mathrm{keV})$ & $12 \mathrm{~m}^{2}(8 \mathrm{keV})$ \\
& $1.0 \mathrm{~m}^{2}(30 \mathrm{keV})$ & $1.2 \mathrm{~m}^{2}(30 \mathrm{keV})$ \\
$\Delta \mathrm{E}$ & $<260 \mathrm{eV}$ & $<200 \mathrm{eV}$ \\
$($ FWHM, @6 keV) & $(200 \mathrm{eV}, 40 \%)^{a}$ & $(160 \mathrm{eV}, 40 \%)^{a}$ \\
FoV (FWHM) & $<60 \mathrm{arcmin}$ & $<30 \mathrm{arcmin}$ \\
Time res. & $10 \mu \mathrm{s}$ & $7 \mu \mathrm{s}$ \\
Dead time & $<1 \%(@ 1 \mathrm{Crab})$ & $<0.5 \%(@ 1 \mathrm{Crab})$ \\
Background flux & $<10 \mathrm{mCrab}$ & $<5 \mathrm{mCrab}$ \\
Max. src flux (steady) & $>0.5 \mathrm{Crab}$ & $>0.5 \mathrm{Crab}$ \\
Max. src flux (peak) & $>15 \mathrm{Crab}$ & $>30 \mathrm{Crab}$ \\
\hline \multicolumn{2}{l}{ : Refers to single-anode events. }
\end{tabular}

\begin{tabular}{|c|c|c|}
\hline \multicolumn{3}{|c|}{ WFM } \\
\hline Parameter & Requirement & Goal \\
\hline Energy range & $2-50 \mathrm{keV}$ & $1-50 \mathrm{keV}$ \\
\hline$\Delta \mathrm{E}(\mathrm{FWHM})$ & $<500 \mathrm{eV}$ & $<300 \mathrm{eV}$ \\
\hline FoV (FWHM) & $\begin{array}{l}50 \% \text { of the sky } \\
\text { accessible to } \\
\text { the LAD }\end{array}$ & $\begin{array}{l}\text { Same as requirements. } \\
\text { Sensitivity improvements } \\
\text { are the prime goal }\end{array}$ \\
\hline Angular res. & $5 \operatorname{arcmin}$ & $3 \operatorname{arcmin}$ \\
\hline Position Accuracy & $1 \operatorname{arcmin}$ & $0.5 \operatorname{arcmin}$ \\
\hline $\begin{array}{l}\text { Sensitivity } \\
(5 \sigma, 50 \mathrm{ks})\end{array}$ & $5 \mathrm{mCrab}$ & $2 \mathrm{mCrab}$ \\
\hline $\begin{array}{l}\text { Sensitivity } \\
(5 \sigma, 1 \mathrm{~s})\end{array}$ & $1 \mathrm{Crab}$ & $0.2 \mathrm{Crab}$ \\
\hline
\end{tabular}

Table 1: Scientific requirements and goals of the LOFT/LAD and LOFT/WFM.

detectors and capillary plate collimators (in the range of $\sim 10 \mathrm{~kg} \mathrm{~m}^{-2}$ compared to $>100 \mathrm{~kg} \mathrm{~m}^{-2}$ of the RXTE/PCA). The basic set-up of the instrument is a set of 6 Detector Panels tiled with 2000 Silicon Drift Detectors (SDDs, heritage of the ALICE experiment at CERN/LHC; Vacchi et al., 1991; Zampa et al., 2010; Campana et al., 2011), which operate in the energy range 2-50 keV and have an energy resolution of $\sim 260 \mathrm{eV}$ (FWHM, at $6 \mathrm{keV}$ ). The modular structure (see Fig. 2) ensures a high level of redundancy and the robustness of the instrument against single units failures. The field of view of the LAD is limited to $\sim 40$ arcmin by X-ray collimators. These are developed by using the technique of micro-capillary plates, the same used for the micro-channel plates: a $3 \mathrm{~mm}$ thick sheet of Lead glass is perforated by a huge number of micro-pores, $\sim 20 \mu \mathrm{m}$ diameter, 4-6 $\mu \mathrm{m}$ wall thickness. The stopping power of Lead in the glass over the large number of walls that off-axis photons need to cross is effective in collimating X-rays below $50 \mathrm{keV}$. In order to accommodate for the internal misalignments of the instrument and for attitude uncertainties, the response of the collimator in the central $\sim 10-15$ arcmin angle is flat (flat-top response) to avoid any spurious modulation of the detected source flux. A summary of the presently established requirements and goals of the LAD are reported in Table 1.

\subsection{The wide field monitor}

The LOFT baseline ${ }^{2}$ WFM is a coded aperture imaging experiment designed on the heritage of the SuperAGILE experiment successfully operating in orbit since 2007 (Feroci et al., 2007). With the $\sim 100 \mu \mathrm{m}$ position resolution provided by its Silicon microstrip detector, SuperAGILE demonstrated the feasibility of a compact, large-area, light, and low-power high resolution Xray imager, with steradian-wide field of view. The LOFT WFM applies the same concept, with improvements provided by the higher performance (low energy threshold and energy resolution) of the Silicon Drift Detectors (SDDs) in place of the Si microstrips. The working principle of the WFM is the classical sky encoding by coded masks and is widely used in space borne instruments (e.g. INTEGRAL, RXTE/ASM, Swift/BAT). The mask shadow recorded by the position-sensitive detector can be deconvolved by using the proper procedures and recover the image of the sky, with an angular resolution given by the ratio between the mask element and the mask-detector distance. By using SDDs with a position resolution $<100 \mu \mathrm{m}$, a coded mask at $\sim 200 \mathrm{~mm}$ provides

\footnotetext{
${ }^{2}$ An alternative solution is also under consideration which implies the usage of double sided Silicon strip detectors; see http://www.isdc.unige.ch/loft/index.php/instruments-on-board-loft.
} 

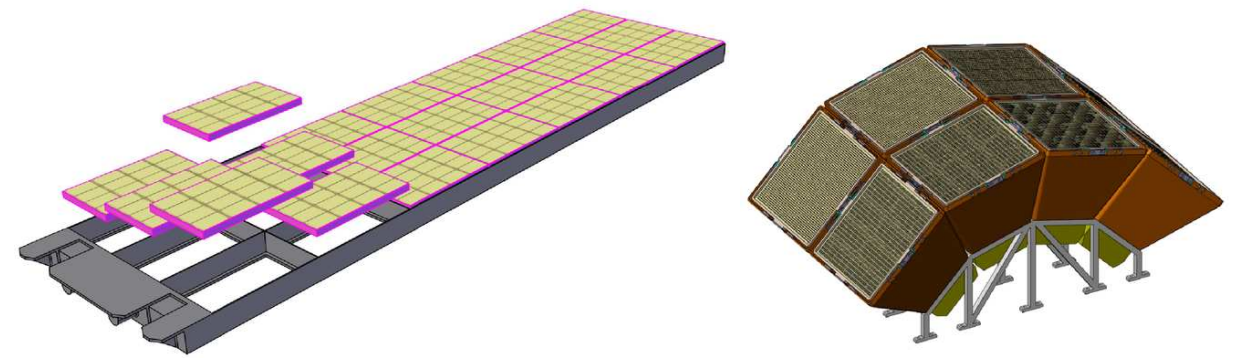

Figure 2: Left panel: Details of the modular structure of one LAD panel (21 modules hosting 16 SDD each). Right panel: The current design of the WFM, comprising 4 units constituted by two co-aligned cameras each.

an angular resolution $<5$ arcmin. As a first approach, each WFM camera can be considered a one-dimensional coded mask imager. This means that after the proper deconvolution is applied to the detector images, the image of a sky region including a single point-like source will appear as a single peak over a flat background. The position of the peak corresponds to the projection of the sky coordinates onto the WFM reference frame. The width of the peak is the point spread function (few arc minutes for the LOFT/WFM). If more than one source is present in the observed sky region, the image will show a corresponding number of individual peaks, whose amplitude will depend on the intensity of the source and on the exposed detector area at that specific sky location. By observing simultaneously the same sky region with two cameras oriented at $90^{\circ}$ to each other (such pair composing one WFM unit), one can derive the precise 2D position of the sources, by intersecting the two orthogonal 1D projections. The overall configuration of the WFM envisages at present a set of 4 units, each comprising 2 co-aligned cameras (see Fig. 2). The 4 units are off-set one to other along one direction in order to provide the maximum coverage of the region of the sky accessible to the LAD (see also Table 2.1).

\section{Conclusions}

LOFT was primarily designed to address the fundamental questions raised by the Cosmic Vision under the "matter under extreme conditions" theme, through the study of the spectral and fast flux variability of compact X-ray sources. The high throughput required by this type of investigation is achieved by an unprecedented large collecting area $\left(\sim 10 \mathrm{~m}^{2}\right.$ at $\left.8 \mathrm{keV}\right)$, coupled with a spectral resolution of $\sim 260 \mathrm{eV}$ (FWHM at $6 \mathrm{keV}$ ). A simulation of the LOFT capabilities is shown in Fig. 3 for two of the main science drivers of the mission: the measurement of the NS mass and radius in Galactic X-ray binaries and the detection of broad iron lines in bright AGNs. The science investigations that will be accessible with LOFT are far too broad to be efficiently summarized here, and we remind the reader to the documentation available on the official LOFT website ${ }^{3}$.

Following the selection of LOFT as one of the four candidate M3 missions for the Cosmic Vision programme in 2011, a further down-selection is expected in 2013. This will bring only one of the candidate missions to a launch in the 2020s.

\footnotetext{
${ }^{3}$ http://www.isdc.unige.ch/loft
} 

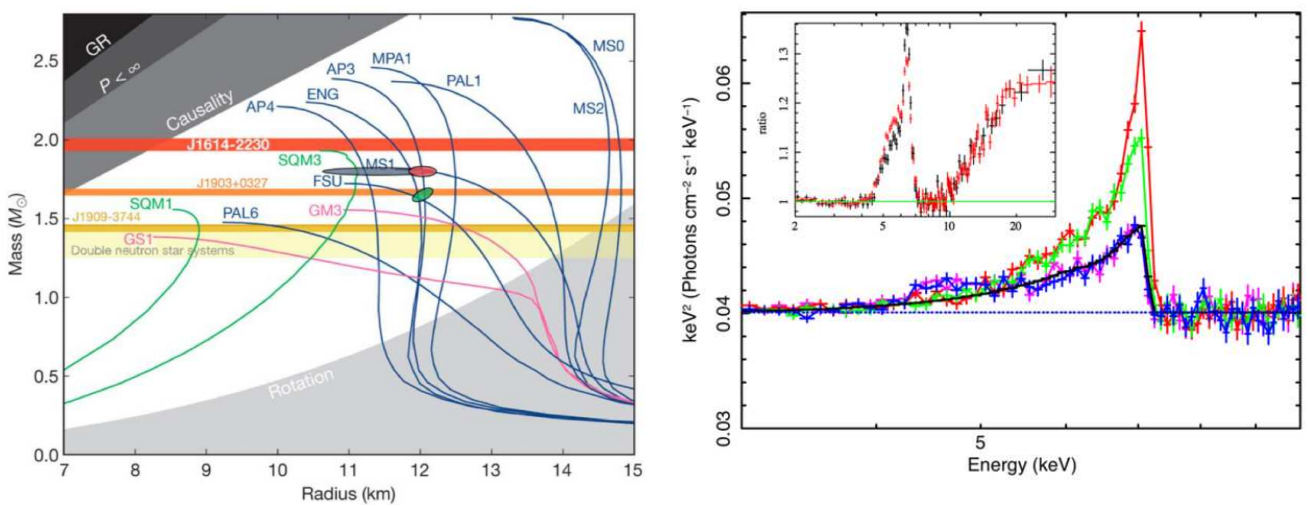

Figure 3: Left panel: Mass-radius curves of representative EOSs for nucleonic (blue), nucleonic plus exotic (pink), and strange quark (green) matter. Horizontal bars represent mass determinations of binary radio pulsars. Regions to the upper left are disallowed by General Relativity and causality. The lower right region is excluded by the highest-frequency pulsar known $(716 \mathrm{~Hz})$. The pulse profile rising phase of a Type I X-ray burst was simulated by assuming the LAD capabilities in a way similar to Strohmayer (2004). The derived $90 \%$ confidence level limits on the NS M and R from observations with LOFT are shown by the green ellipse. The uncertainty is $<5 \%$ on both $\mathrm{M}$ and $\mathrm{R}$. The M-R constraint derived from fitting simulated pulse profiles of SAX J1808.4-3658, the first millisecond $\mathrm{X}$-ray pulsar discovered $(401 \mathrm{~Hz})$, are shown by a gray ellipse. The same technique applied to simulations of the signal of the first eclipsing millisecond X-ray pulsars, Swift J1749.4-2807 (518 Hz), gives in a much smaller uncertainty region (red); this is mainly due to the higher system inclination. Right panel: Simulated LOFT/LAD spectra of a $3 \mathrm{mCrab}(2-10 \mathrm{keV})$ AGN. The lower plot shows the broad relativistic Fe line produced in the innermost region of the accretion disc extending down to the marginally stable for an almost-maximally rotating $\mathrm{BH}(a=0.99)$ with mass of $3.6 \times 10^{6} \mathrm{M} \odot$, viewed at an inclination of $45^{\circ}$. The plot shows the variable $\mathrm{Fe} \mathrm{K}$ feature produced by an orbiting spot at $r=10 \mathrm{GM} / \mathrm{c}^{2}$ on the disk surface that is illuminated by a flare. The orbital period is $4 \mathrm{ks}$ and the total exposure is $16 \mathrm{ks}$. LOFT/LAD will track the line variation on $1 \mathrm{ks}$ time scale, thus allowing a determination of the orbital period through measurements at four different orbital phases over four cycles. The inset shows two $10 \mathrm{ks}$ spectra from simulated high (6 mCrab in 2-10 keV) and low (2 mCrab) flux states LOFT/LAD observations of MCG-6-30-15. Thanks to the wide energy band and the large effective area above $10 \mathrm{keV}$, the variation of the reflection hump can be measured with $2 \%$ accuracy.

\section{References}

Campana, R. et al. 2011, NIMPA, 633, 22

Feroci, M. et al. 2011, ExA, in press (arXiv:1107.0436).

Feroci, M. et al. 2007, NIMPA, 581, 728.

Jahoda, K. et al., SPIE 1996, 2808, 59.

Psaltis, D., in Living Reviews in Relativity 2008, 11, 9.

Stella, L., in Physics of Relativistic Objects in Compact Binaries, Springer Netherlands.

Strohmayer, E.T. 2004, AIPC, 714, 245.

Strohmayer, E.T., Watts, A.L. 2006, ApJ, 653, 593.

Vacchi, A. et al. 1991, Nucl. Meth. Instr. A, 306, 187.

van Der Klis, M., in Compact Stellar X-ray Sources 2006, Cambridge University Press.

Zampa, G., et al. 2010, SPIE, 7732, 146 